\title{
On the Motion of a Projectile in the Atmosphere
}

\author{
By Vi-Cheng LiU, Ann Arbor, Michigan, USA ${ }^{1}$ )
}

\begin{abstract}
The equation of rectilinear motion of a projectile which moves in an atmosphere, of which the density decreases exponentially with the altitude, is solved. It is found that the velocity of the projectile can be expressed explicitly in terms of confluent hypergeometric functions. This theory is applied to treat two specific problems: (1) the flight analysis of a sounding rocket during the freeflight period and (2) the calculation of ambient temperature from the trajectory of a spherical projectile.
\end{abstract}

\section{Introduction}

The theory of flight of a ballistic projectile in a resisting medium dates back to NEwToN. In the meantime, various approximations for the flight parameters which are involved in the equation of motion have been used [1] $\left.{ }^{2}\right)$. The most important ones are the aerodynamic-drag coefficient of the projectile and the ambient-air density. The former is usually considered as functions of Mach number, the latter as functions of the altitude.

The theory of flight of a sounding rocket during the free-flight period deals essentially with the same problem as that of a ballistic projectile. The only difference is that the air-density factor is more critical because of the extreme altitude attained by the former; hence, a more realistic approximation to the ambient-air density is necessary. In view of the multiplicity of parameters involved, the equation of motion of the projectile (or rocket) is generally treated. either by a laborious method of step-wise integration or by the analog computer technique.

A recent experiment [2], in which a falling sphere is used as an ambienttemperature probe in the upper-atmosphere measurements, stimulates new interest to the trajectory problem. Measured trajectory data of a spherical projectile are used to determine the ambient-air density, from which the ambient-air temperature can be derived.

In this note, new approximations to the aerodynamic-drag coefficient and ambient-air density, which are believed to be more accurate than previous representations [1], are introduced. Particle drag, which is caused by the presence of aerosols in the atmosphere, is allowed in the present analysis. This, of course, warrants consideration only in some special cases.

It is found that the projectile velocity is expressible explicitly in terms of confluent hypergeometric functions. The significance of this result, which is believed to be new, is twofold: (1) In treating 'direct' problems, for instance, like the trajectory analysis of a sounding rocket, the present theory can be used to save the labor of step-wise integration if tabulation of the particular confluent hypergeometric function is available. (2) In the case of an 'indirect' problem such as the computation of the ambient temperature from a falling-sphere trajectory, it can be applied with advantage because of the elimination of the numerical differentiation process which is involved in the original theory [2].

1) High Altitude Engineering Laboratory, University of Michigan.

2) Numbers in brackets refir to References, page 81. 


\section{Significant Forces}

The word 'projectile' is used here referring to any axially symmetric body in free flight. We restrict our discussion to the case with negligible spin and yaw.

The force system acting on a projectile includes: (1) the gravity $m g$; (2) the buoyancy $\varrho g V$, where $V$ represents the volume displaced by the projectile, and $\varrho$ the ambient-air density; (3) the aerodynamic drag $D$; (4) particle drag $d$, which is caused by the presence of aerosols in the atmosphere; and (5) the inertial force $(m+\Delta m) d v / d t$, where $\Delta m$, Munz's apparent additional mass, is equal to one half the mass of air displaced by a spherical projectile; and $d v / d t$, the projectile acceleration.

The buoyancy and the inertial force of the apparent additional mass are significant only for projectiles of the balloon type. Though, under sea-level conditions, the particle drag due to water droplets, for instance, may be insignificant when compared with the aerodynamic drag, it is conceivable, however, that in the cumulus clouds at high altitudes, the drag due to water droplets may become significant. While the water-droplet content depends primarily on the ambient temperature, the ambient-air density decreases with the altitude exponentially.

It is not intended here for us to become involved in detailed computation of the particle drag. Rather, we shall investigate its effect on the projectile motion since the particle-drag term in the equation of motion follows a functional dependence upon the altitude, which is, in general, different from that of the aerodynamic drag. As a first approximation, the particle drag is prescribed as $d=C_{d} \sigma A v^{2} / 2$, according to the Newtonian concept of drag. The particles are assumed to be mass points at rest; hence, $C_{d}$ should depend only on the position and shape of the projectile and be independent of its size $A$ und velocity $v$. The particle concentration is represented by $\sigma$.

\section{Approximate Representation of the Aerodynamic-Drag Coefficient}

It can be demonstrated with a dimensional analysis that the aerodynamicdrag coefficient, defined as $C_{D}=D /\left(\varrho V^{2} A / 2\right)$, for geometrically similar projectiles depends primarily upon the Mach number and Reynolds number. For projectiles moving at high speeds, the dependence of $C_{D}$ on the Reynolds number is much less significant than that on the Mach number except in cases for which the Reynolds numbers are low, such as those at the high altitudes. In those cases, however, the aerodynamic-drag force is usually negligible as compared to the gravity.

For a specific projectile, the variation of $C_{D}$ with Mach number $M$, in general, shows distinctly different characteristics in the three Mach number ranges: (1) subsonic, (2) transonic, and (3) supersonic. Qualitatively speaking, $C_{D}(M)$ starts with roughly constant values (for different projectiles) then increases gradually as transonic range is approached. During the transonic range it increases until the supersonic range begins. $C_{D}(M)$ in the supersonic range decreases with a gradient which is very large at first and gradually diminishes to a small value.

Reliable values of $C_{D}$ still rely mostly on measurements from model tests. Theoretical consideration may, however, serve as a guide to the choice of empirical functions for prescribing $C_{D}$. 
Consider separately the compressibility effect in the subsonic flow for slender and round bodies. In the former case, to account for the compressibility effect on the pressure coefficient, either the Prandtl-Glauert factor $\left(1-M^{2}\right)^{-1 / 2}$ or a modified version of it (for instance, the Karman-Tsien formula or GoETHERT's generalized Prandtl-Glauert formula) can be used. These factors, when expanded in a power series of $M$, can be expressed in the form

$$
C_{0}+C_{2} M^{2}+\cdots
$$

after neglecting higher-order terms. In the latter case, as an index of the compressibility on the drag coefficient of a round body, the impact-pressure coefficient at the stagnation point can be shown to be

$$
C_{p}=1+\frac{M^{2}}{4}+\cdots .
$$

It has also been shown by measurements that the compressibility effect on the skin-friction coefficient and the coefficient of drag due to interference between body and fins can be considered as proportional to the powers of the PrandtlGlauert factor.

A large fraction of the drag force on a projectile in the supersonic flow are attributed to the frontal shock wave and the base pressure. For a typical body like a cylinder with a nose cone, the drag coefficient can be shown to be approximately of the form

$$
C_{0}+C_{3} M^{-2}
$$

The validity of the above expression is expected to extend to the hypersonic range [3]. Relatively less is known concerning the theory of drag in a transonic flow, which is a mixture of subsonic and supersonic flows. It is therefore suggested to use

$$
C_{D}=C_{0}+C_{2} M^{2}+C_{3} M^{-2}
$$

as a general expression for approximating the drag coefficient of a projectile in the following analysis. $C_{0}, C_{2}$, and $C_{3}$ in (3.4) are empirical constants to be prescribed for each Mach-number range.

\section{Equation of Rectilinear Motion of a Projectile}

Consider a nonspinning projectile which ascends at zero angle of yaw in a stationary atmosphere. By collecting terms of forces which have been discussed in Section 2, we obtain the equation governing the rectilinear motion of a projectile along the vertical axis $y$.

$$
\begin{aligned}
\frac{1}{2}\left(C_{0}+C_{2} M^{2}+\right. & \left.C_{3} M^{-2}\right) A \varrho v^{2} \\
& +\frac{1}{2} C_{d} A \sigma v^{2}-\varrho g V+m g+(m+\Delta m) v \frac{d v}{d y}=0 .
\end{aligned}
$$

For an isothermal atmosphere with temperature $T$ in altitude interval $\left(y-y_{0}\right)$, the ambient-air density can be expressed as $\varrho=\varrho_{0} \exp \left[-\left(y-y_{0}\right) / H\right]$ where $H$, the scale height, is defined as $k T / m_{1} g\left(k=\right.$ BoLTZMANN's constant; $m_{1}=$ mean molecular mass of air). 
To simplify equation (4.1), we introduce

$$
Z=M^{2}=(\gamma g H)^{-1} v^{2}, \quad x=\exp -\frac{y-y_{0}}{H}, \quad \text { and } \varrho=\varrho_{0} x,
$$

where $\gamma$ is the ratio of specific heats of air, and obtain

where

$$
\frac{d Z}{d x}=C_{2} \alpha Z^{2}+\left(C_{0} \alpha+\frac{\beta}{x}\right) Z+\left(C_{3} \alpha-\delta+\frac{\varepsilon}{x}\right),
$$

$$
\left.\begin{array}{l}
\alpha=A \varrho_{0} H(m+\Delta m)^{-1}, \quad \beta=C_{d} A \sigma H(m+\Delta m)^{-1}, \\
\delta=2 \varrho_{0} V \gamma^{-1}(m+\Delta m)^{-1}, \quad \varepsilon=2 m \gamma^{-1}(m+\Delta m)^{-1} .
\end{array}\right\}
$$

Equation (4.3) is nonlinear and of the Riccati type. It can be transformed through use of the substitution

into a linear equation

$$
u=\exp \left[-\int \alpha C_{2} Z d x\right]
$$

$$
\frac{d^{2} u}{d \tau^{2}}-\left(\alpha C_{0}+\frac{\beta}{x}\right) \frac{d u}{d x}+\alpha C_{2}\left(\alpha C_{3}-\delta+\frac{\varepsilon}{x}\right) u=0
$$

Equation (4.6), which has a regular singularity at $x=0$ and an irregular singularity at $x=\infty$, can be converted into the canonical form

with

$$
\xi \frac{d^{2} \varphi}{d \xi^{2}}+(b-\xi) \frac{d \varphi}{d \xi}-a \varphi=0
$$

$$
\left.\begin{array}{c}
\xi=2 \Omega x, \quad \Omega^{2}=\frac{\alpha^{2} C_{0}^{2}}{4}+\alpha C_{2}\left(\delta-\alpha C_{3}\right), \quad \varphi=u \exp \left[\Omega-\frac{1}{2} \alpha C_{0}\right] \mathcal{X}^{\prime} \\
b=-\beta, \quad a=\frac{1}{2}\left(\frac{1}{2} \propto C_{0} \beta-\alpha C_{2} \varepsilon-\beta \Omega\right) \Omega^{-1} .
\end{array}\right\}
$$

One of the fundamental solutions of (4.7) is the confluent hypergeometric function [4]

where

$$
\varphi(a, b, \xi)={ }_{1} F_{1}(a, b, \xi)=\sum_{n=0}^{\infty} \frac{(a)_{n} \xi^{n}}{(b)_{n} n !},
$$

The other one is

$$
\begin{aligned}
(a)_{n} & =a(a+1) \cdots(a+n-1) \quad(n \geqq 1), \\
(a)_{0} & =1 .
\end{aligned}
$$

$$
\xi^{1-b}{ }_{1} F_{1}(1+a-b, 2-b ; \xi)
$$

provided $b$ is not an integer. The general solution of (4.7) with nonintegral $b$ may be written as

$$
\Phi=B_{1} F_{1}(a, b ; \xi)+C \xi^{1-b}{ }_{1} F_{1}(1+a-b, 2-b ; \xi),
$$

where $B$ and $C$ are arbitrary constants. Confluent hypergeometric functions have been fairly extensively tabulated $([5],[6],[7])$.

In view of (4.2), (4.5), (4.8), and (4.11), we obtain

$$
v^{2}=\frac{2 \gamma g H \Omega}{\alpha C_{2}}\left[\frac{1}{2}-\frac{\alpha C_{0}}{4 \Omega}-\frac{1}{\Phi} \times \frac{d \Phi}{d \xi}\right] .
$$


Because of the term $(d \Phi / d \xi) / \Phi$, one of the arbitrary constants of $\Phi$ as given in equation (4.11) will be eliminated in equation (4.12) for $v^{2}$. Since equation (4.3) is of the first order, there is only one constant of integration. This is consistent with the physical aspect of the problem. The constant of integration is to be determined by the use of the boundary condition of the projectile trajectory.

Consider the restriction on $b$ in (4.11). Since $b=-\beta$, where $\beta$ is defined as $C_{d} A \sigma H(m+\Delta m)^{-1}$, which, in general, is less than unity, the limitation on the values of $b$ is not significant as far as the present analysis is concerned. The only notable exception is the case $b=0$, which corresponds to the problem when the particle drag is null.

Equation (4.7) with $b=0$ has been studied by BATEMAN [8]. The solution of (4. 7) for this particular case is known as BATEMAN's $k$-function. The general solution of equation (4.7), when $b=0$, is bounded in the neighborhood of $\xi=0^{3}$ ).

It is to be noted that when $C_{2}=0$, equation (4.3) becomes a linear equation of the first order, which can be integrated immediately in terms of elementary functions [9].

It is also to be noted that the equation of motion of a projectile with the assumption $C_{D} \sim M^{-1}$ (a satisfactory approximation for the wave drag of airfoil sections in hypersonic flows) and $\beta=0$ is again a linear equation of the first order if $v$ and $t$ are used as a dependent and an independent variable, respectively [10].

It is interesting to note that the function $p$ in equation (4.7) can be expressed in terms of Bessel functions provided that the condition $2 C_{2} \varepsilon=C_{0} \beta$ is fulfilled. Other special cases of the confluent hypergeometric function will be obtained for the solution $\varphi$ when values of the parameters $b$ and $a$ in equation (4.7) fulfill appropriate restrictions in each individual case.

\section{Engineering Applications}

The result of the present analysis may be applied to the following problems:

\subsection{Flight Analysis of a Sounding Rocket}

A rocket which carries sounding instruments to high altitudes for measuring the upper atmosphere is called a sounding rocket. It is usually launched vertically upward to gain the utmost peak altitude. In view of the simplicity of the designed trajectory, it is generally equipped with the least guidance. As a first approximation, it is plausible to assume that a sounding rocket with normal performance moves rectilinearly at zero angle of yaw. Hence, the present analysis can be applied in the free-flight period.

For a solid-propellant rocket, which usually has large jet-propulsive thrust of a short duration, we can estimate the burnout velocity and altitude by neglecting the aerodynamic drag in comparison to the forward thrust. The classical method is to integrate the equation of motion of a rocket with mass $m$ as a function of time $t$ :

$$
C \cdot \frac{d m}{d t}+m\left(\frac{d v}{d t}+g\right)=0
$$

where $C$ is the constant exhaust velocity. This leads to

$$
v_{\imath}=-C \ln \frac{m_{0}}{m_{0}}-g\left(t_{t}-t_{0}\right),
$$

3) This is pointed out to the author by Professor Robert C. F. Bartels. 


$$
y_{b}=-C \int_{t_{0}}^{t_{b}}\left(\ln \frac{m_{b}}{m_{0}}\right) d t-\frac{1}{2} g\left(t_{b}-t_{0}\right)^{2}
$$

where subscripts 0 and $b$ denote initial and burnout condition, respectively. The above calculations can be improved through an iterative process in regard to the aerodynamic-drag correction.

\subsection{Ambient Temperature Computation from Trajectory Data of a Falling Sphere}

The idea of using a free-falling sphere, of which the trajectory can be accurately measured, to probe the upper atmosphere for the purpose of measuring the ambient temperature was originally conceived by Jones [2]. This was carried out by incorporating a miniature Doppler receiver-transmitter in an inflated sphere, which was released from a sounding rocket near its peak altitude. The sphere trajectory is determined by a DOVAP system [11], which consists of a transmitter and an array of receivers on the ground.

In an alternative scheme, as proposed by the author, the ground stations of transmitters and receivers are replaced by a mobile station which has a transmitter and a receiver just like those used in the DOVAP system except in miniature sizes. The mobile station is incorporated in a body, with negligible drag-to-weight ratio, which is to be released simultaneously with the sphere from the rocket. With this scheme, the velocity of the sphere, relative to an apparently null-drag trajectory or pseudo-vacuum trajectory, is obtained from Doppler cycles which are to be telemetered from the mobile station and recorded along a time scale. It is assumed that both the mobile station and the sphere fall vertically with no relative tumble.

One serious drawback of the falling sphere method is the need of numerical differentiation of the velocity data in the process of computing ambient-air density according to the original theory [2]. As it is known, numerical differentiation is a notoriously inaccurate process because it exaggerates the irregularities of the numerical function whose derivatives are to be determined. This process can be avoided if the present theory of projectile trajectory is applied in step-wise calculation of the scale height $H$, which is proportional to the ambientair temperature $T$.

\section{Acknowledgment}

This work was carried out in the Department of Aeronautical Engineering as a research project on atmospheric phenomena at high altitudes under the sponsorship of the US Army Signal Corps in a contract with the Engineering Research Institute of the University of Michigan. The author is indebted to Mr. L. M. JoNES for interest and encouragement.

\section{REFERENCES}

[1] E. J. McShane et al., Exterior Ballistics (University of Denver Press, Denver 1953), p. 742.

[2] F. L. Bartman, Falling Sphere Method for Upper Air Density and Temperature, in: Rocket Exploration of the Upper Atmosphere, Special Supplement, vol. 1, to J. atmos. terr. Phys. (Pergamon Press, Ltd., London 1954), p. 98. 
[3] T. von Karman, Proc. first nat. Congr. appl. Mech. 1952, 673.

[4] A. ERDÉLxi, Higher Transcendental Functions, vol. I (Bateman Manuscript Project) (McGraw-Hill Book Co., Inc., New York 1953), p. 252.

[5] British Association for Advances in Science, Section A: Mathematical Tables (Oxford 1926, Leeds 1927).

[6] E. Jahnke et al., Tables of Functions (Dover Publications, Inc., New York 1945), p. 275.

[7] A. FLETCHER et al., Index of Mathematical Tables (Cambridge University Press, Cambridge 1946).

[8] H. Bateman, Trans. Amer. math. Soc. 33, 817 (1931).

[9] H. G. L. Krause, Proc. 4th astronaut. internat. Congr. (Zurich 1953).

[10] M. S. Jones, Jet Propulsion 25, 531 (1955).

[11] D. Hoffleit, Sci. Mon., N. Y. 48, 172 (1.949).

\section{Zusammenfassung}

Die Bewegungsgleichung eines vertikal ansteigenden Geschosses wird gelöst für den. Fall, dass die Dichte der Atmosphäre exponentiell mit der Höhe abnimmt. Die Geschwindigkeit des Projektils kann dabei in konfluenten hypergeometrischen Funktionen ausgedrückt werden. Die Theorie wird hierauf auf zwei spezifische Probleme angewendet: 1. Fluganalyse einer Vertikalrakete während der Zeit des schubfreien Fluges und 2. Berechnung der Umgebungstemperatur der Bahn eines sphärischen Projektils.

(Received: July 30, 1956.)

Buchbesprechungen - Book Reviews - Notices bibliographiques

Numerische Behandlung von Differentialgleichungen. Von L. Collatz. Zweite, neubearbeitete Auflage (Springer-Verlag, Berlin 1955 [Grundlehren der mathematischen Wissenschaften, Band 60]). 526 S., 118 Abb.; DM 56.-/59.60.

Die zweite Auflage des Handbuches über numerische Lösungsmethoden für Differentialgleichungen unterscheidet sich von der ersten Auflage vor allem durch Aufnahme der in der Zwischenzeit gewonnenen neuen Erkenntnisse, insbesondere der Instabilitätsphänomene bei numerischer Integration. Ausserdem wurde die Einteilung des Werkes etwas verändert, indem alle mathematischen Grundlagen im 1. Kapitel zusammengefasst sind. Es folgen wie früher: If. Anfangswertprobleme; III. Randwertprobleme bei gewöhnlichen Differentialgleichungen; IV. Anfangs- und Anfangsrandwertaufgaben bei partiellen Differentialgleichungen; V. Elliptische partielle Differentialgleichungen; VI. Integral- und Funktionalgleichungen.

H. Rutishauser

Mécanique vibratoire. Par RoBERx MAZET (Librairie polytechnique $\mathrm{Ch}$. Béranger, Paris 1955). 280 pp., 159 fig.; relié fr.fr. 4975,--

De nombreux ouvrages ont été publiés sur la Mécanique vibratoire. Celui de M. Mazet, professeur à la Faculté des Sciences de Poitiers, reproduit les leçons données par cet auteur à l'Ecole nationale supérieure de l'Aéronautique, de 1950 à 1954.

Il s'agit avant tout de l'étude des systèmes mécaniques dits "linéaires", dont l'évolution relève, avec une précision suffisante, d'équations différentielles 